\title{
Single molecular-excitation propagation in one-dimensional optical lattices
}

\section{Propagación de una excitación molecular en redes ópticas unidimensionales}

\author{
Vanessa Carolina Olaya-Agudelo ${ }^{1}$, Karen Rodríguez-Ramírez ${ }^{1}{ }^{*}$ \\ 1. Departamento de Física, Universidad del Valle, A.A. 25360, Cali, Colombia \\ ${ }^{(*)}$ E-mail: karem.c.rodriguez@correounivalle.edu.co
}

Received: 16/04/2018 Accepted: 06/03/2019

DOI: $10.7149 /$ OPA.52.2.50310

\begin{abstract}
:
We study a LiCs strongly-interacting molecular gas loaded on an one-dimensional optical lattice keeping one particle per site. The molecules are in the lowest electronic and vibrational $(v=0)$ state, $\mathrm{X}^{1} \Sigma$. Here, coherent transfers of rotational excitations $(\mathrm{N}=\{0,1\})$ along the lattice are shown for several lattice sizes and initial conditions. Due to the large intermolecular distance and low filling, the dipoledipole interaction in the nearest-neighbor approximation governs the dynamics of the rotational excitations. For low field strengths, the full set of internal rotational projections must be taken into account. The generated von Neumann entanglement entropy throughout the dynamics of the energy transfer are also presented. A monotonic growth for short times and a saturation towards the steady regime is observed, showing the potential of these molecular systems to be used in quantum information protocols. The numerical simulations are performed by means of the Time-Evolving Block Decimation algorithm based on the Matrix Product State formalism and the Susuki-Trotter decomposition.
\end{abstract}

Key words: Atomic and molecular physics, coherent optical effects, ultracold collisions, electric dipole moments, polar molecules.

\section{RESUMEN:}

El presente trabajo estudia un gas reticular unidimensional de moléculas LiCs fuertemente interactuantes, con una molécula por sitio, en el estado electrónico y vibracional más bajo $(\nu=0), X^{1} \Sigma$. Se muestran transferencias coherentes de excitaciones rotacionales $(\mathrm{N}=\{0,1\})$ a lo largo de la red para varios tamaños de red y condiciones iniciales, donde la interacción dipolo-dipolo rige la dinámica. Se muestra que para intensidades bajas del campo se debe considerar el conjunto completo de los estados rotacionales internos. Se presenta la entropía de entrelazamiento de von Neumann observándose un crecimiento monótono para tiempos cortos y una saturación hacia el régimen estacionario para tiempos largos, evidenciando el potencial de estos sistemas para ser utilizados en protocolos de información cuántica. Las simulaciones numéricas se realizan por medio del algoritmo de decimación de bloques en la evolución del tiempo (TEBD) basado en el formalismo del estado productos de matrices (MPS) y la descomposición de Susuki-Trotter.

Palabras clave: Física atómica y molecular, efectos ópticos coherentes, colisiones ultra frías, momentos dipolares eléctricos, moléculas polares.

\section{REFERENCES AND LINKS / REFERENCIAS Y ENLACES}

[1] J. J. Hudson, B. E. Sauer, M. R. Tarbutt, E. A. Hinds, "Measurement of the electron electric dipole moment using YbF molecules," Phys. Rev. Lett., 89, 023003 (2002).

[2] T. Zelevinsky, S. Kotochigova, J. Ye, "Precision test of mass-ratio variations with lattice confined ultracold molecules,” Phys. Rev. Lett. 100, 043201, (2008). 
[3] D. DeMille, S. Sainis, J. Sage, T. Bergeman, S. Kotochigova, E. Tiesinga, "Enhanced sensitivity to variation of me /mp in molecular spectra," Phys. Rev. Lett. 100, 043202, (2008).

[4] R. V. Krems, "Molecules near absolute zero and external field control of atomic and molecular dynamics," Int. Rev. Phys. Chem. 24, 99-118 (2005).

[5] N. Balakrishnan, "Perspective: Ultracold molecules and the dawn of cold controlled chemistry," J. Chem. Phys 145, 15090 (2016).

[6] D. DeMille, “Quantum computation with trapped polar molecules,” Phys. Rev. Lett. 88, 067901 (2002).

[7] P. Rabl, P. Zoller, "Molecular dipolar crystals as high-fidelity quantum memory for hybrid quantum computing," Phys. Rev. A 76, 042308 (2007).

[8] K. Rodriguez, S. R. Manmana, M. Rigol, R. M. Noack, A. Muramatsu, "Coherent matter waves emerging from mott-insulators," New J. Phys. 8, 169 (2006).

[9] K. Rodríguez, A. Argüelles, M. Colomé-Tatché, T. Vekua, L. Santos, “Mott-insulator phases of spin-3/2 fermions in the presence of quadratic Zeeman coupling," Phys. Rev. Lett. 105, 050402 (2010).

[10] K. Rodríguez, A. Argüelles, A. K. Kolezhuk, L. Santos, T. Vekua, "Field-induced phase transitions of repulsive spin-1 bosons in optical lattices," Phys. Rev. Lett. 106, 105302 (2011).

[11] A. M. Kaufman, B. J. Lester, C. A. Regal, "Cooling a single atom in an optical tweezer to its quantum ground state," Phys. Rev. X 2, 041014 (2012).

[12] J. D. Thompson, T. G. Tiecke, A. S. Zibrov, V. Vuletíc, M. D. Lukin, “Coherence and Raman sideband cooling of a single atom in an optical tweezer," Phys. Rev. Lett. 110,133001 (2013).

[13] A. Do,Caj, M. L. Wall, R. Mukherjee, K. R. A. Hazzard, "Ultracold nonreactive molecules in an optical lattice: Connecting chemistry to many-body physics," Phys. Rev. Lett. 116, 135301 (2016).

[14] D. Jin, J. Ye, "Polar molecules in the quantum regime," Phys. Today 64, 27 (2011).

[15] M. Lemeshko, R. V. Krems, J. M. Doyle, S. Kais, "Manipulation of molecules with electromagnetic fields," Mol. Phys. 111, 1648-1682 (2013).

[16] T. Köhler, K. Goral, P. S. Julienne, "Production of cold molecules via magnetically tunable feshbach resonances,” Rev. Mod. Phys. 78, 1311-1361 (2006).

[17] Y. V. Suleimanov, R. V. Krems, "Efficient numerical method for locating Feshbach resonances of ultracold molecules in external fields," J. Chem. Phys. 134, 014101 (2011).

[18] K. M. Jones, E. Tiesinga, P. D. Lett, P. S. Julienne, "Ultracold photoassociation spectroscopy: Long-range molecules and atomic scattering," Rev. Mod. Phys. 78, 483-535 (2006).

[19] A. J. Kerman, J. M. Sage, S. Sainis, T. Bergeman, and D. DeMille, "Production of ultracold, polar RbCs molecules via photoassociation,” Phys. Rev. Lett. 92, p. 033004, Jan 2004.

[20] J. M. Sage, S. Sainis, T. Bergeman, and D. DeMille, "Optical production of ultracold polar molecules," Phys. Rev. Lett. 94, 203001 (2005).

[21] B. E. Londono, J. Mahecha, E. Luc-Koenig, A. Crubellier, "Lifetime of vibrational levels from Fourier grid calculations: RbCs example," Phys. Chem. Chem. Phys. 13, 18738-18754 (2011).

[22] F. Lang, K. Winkler, C. Strauss, R. Grimm, J. H. Denschlag, "Ultracold triplet molecules in the rovibrational ground state," Phys. Rev. Lett. 101, 33005 (2008).

[23] K. Bergmann, H. Theuer, B. W. Shore, "Coherent population transfer among quantum states of atoms and molecules," Rev. Mod. Phys. 70, 1003-1025, (1998).

[24] P. K. Molony, P. D. Gregory, A. Kumar, C. R. LeSueur, J. M. Hutson, S. L. Cornish, "Production of ultracold 87Rb133Cs in the absolute ground state: Complete characterisation of the stimulated Raman adiabatic passage transfer," Chem. Phys. Chem 17, 3811-3817 (2016).

[25] S. A. Moses, J. P. Covey, M. T. Miecnikowski, D. S. Jin, J. Ye, "New frontiers for quantum gases of polar molecules," Nat. Phys. 13, 13-20 (2017).

[26] J. Deiglmayr, A. Grochola, M. Repp, K. Mörtlbauer, C. Glück, J. Lange, O. Dulieu, R. Wester, M. Weidemüller, "Formation of ultracold polar molecules in the rovibrational ground state," Phys. Rev. Lett. 101, 33004 (2008).

[27] F. Herrera, M. Litinskaya, R. V. Krems, "Tunable disorder in a crystal of cold polar molecules," Phys. Rev. A 82, 033428 (2010).

[28] V. Agranovich, Excitations in Organic Solids. Oxford University Press (2008). 
[29] L. D. Carr, D. DeMille, R. V. Krems, J. Ye, "Cold and ultracold molecules: science, technology and applications," New J. Phys. 11, 055049 (2009).

[30] R. Krems, B. Friedrich, W. Stwalley, Cold Molecules: Theory, Experiments, Applications. Taylor and Francis (2009).

[31] V. R. N. Zare, Angular Momentum: Understanding Spatial Aspects in chemistry and Physics. WileyVCH Verlag GmbH \& Co. KGaA (1988).

[32] E. Schmidt, (German) "Zur theorie der linearen und nichtlinearen integralgleichungen. i. teil: Entwicklung willkrlicher funktionen nach systemen vorgeschriebener," Mathematische Annalen 63, 433-476 (1907).

[33] G. Vidal, "Efficient classical simulation of slightly entangled quantum computations," Phys. Rev. Lett. 91, 147902 (2003).

[34] G. Vidal, "Efficient simulation of one-dimensional quantum many-body systems," Phys. Rev. Lett. 93, 040502 (2004).

[35] F. Verstraete, J. J. Garcia-Ripoll, J. I. Cirac, "Matrix product density operators: Simulation of finitetemperature and dissipative systems," Phys. Rev. Lett. 93, 207204 (2004).

[36] F. Verstraete, D. Porras, J. I. Cirac, "Density matrix renormalization group and periodic boundary conditions: A quantum information perspective," Phys. Rev. Lett. 93, 227205 (2004).

[37] M. Suzuki, "Fractal decomposition of exponential operators with applications to many-body theories and Monte Carlo simulations," Phys. Lett. A 146, 319- 323 (1990).

[38] M. Aymar, 0. Dulieu, "Calculation of accurate permanent dipole moments of the lowest $\sigma+1,3$ states of heteronuclear alkali dimers using extended basis sets," J. Chem. Phys. 122, 204302 (2005).

\section{Introduction}

The new era of cold quantum matter involving ultracold and quantum degenerate molecular gases is currently the focus of interest within atomic physics, molecular physics and physical chemistry communities. The enormous control and high tunability of cold and ultracold molecular systems make them suitable to study a broad application spectra in diverse fields, from precision measurement and highresolution spectroscopy [1-3] to ultracold chemistry [4,5], quantum information processing [6] and quantum computing [7].

Cold lattice gases, either atomic or molecular, are often proposed as quantum simulators mimicking, for instance, the Hubbard [8] and Heisenberg $[9,10]$ models, serving as a way to develop new experimental, theoretical and numerical tools. The production of ultracold ground-state molecules opens the path to derive effective many-body models for nonreactive molecules in tight traps like optical tweezers $[11,12]$ and optical lattices [13]. Arranging polar molecules in optical standing-wave trapping-potentials, new opportunities are expected due to the neighboring sites dipole-dipole interaction; in contrast, atomic lattice gases exhibits only on-site interaction. Besides the long-range character of the dipolar interaction, we exploit its anisotropy which can be manipulated applying external electric fields [14,15].

The successful association of two pre-cooled atoms to form molecules has been achieved using Feshbach resonances [16,17] and photoassociation [18]. Due to the atom-atom association mechanism, it has been shown experimentally $[19,20]$ and numerically $[21]$ that photoassociation in a laser-cooled mixture of 85Rb and $133 \mathrm{Cs}$ atoms, followed by radiative stabilization, populates preferentially $3 \Sigma+$ levels with low binding energy. Hence, the triplet-rovibrational excited state has higher probability to be populated [22] and a further technique known as stimulated Raman adiabatic passage or STIRAP [23,24] must be implemented to achieve the singlet-rovibrational ground state. Although this technique is experimentally complex, it is highly efficient and state-selective. Nevertheless, it can only be applied once per experimental realization, limiting the ultimate number of ground state molecules.

It is of great interest controlling time-evolution among specific discrete quantum states. These individual processes are the basis to understand the reaction rate of chemical reactions [25]. Therefore, in the present work we analyze the coherent population transfer of $\mathrm{X}^{1} \Sigma$ molecules due to the dipole-dipole interaction in their ground and first excited rotational levels as a function of an external electric field [26]. Pursuing this aim, the molecular gas is loaded on a one-dimensional optical lattice and the gas is prepared in the stronglycorrelated Mott-insulator regime. 
This document is divided as follows: in sect. 2 we describe both the model of the molecular lattice system and the implemented numerical method. Section 3 is devoted to the single molecular excitation dynamics in presence of an external DC electric field. The entanglement generated throughout the evolution of the excitations is presented in sect. 4. Finally, in sect. 5 the conclusions are included.

\section{Model and method description}

\section{2.a. Molecular Hamiltonian in the Mott-insulator regime}

We consider an optical lattice filled with X1 $\Sigma$ polar molecules (LiCs molecules), which are in the electronic and vibrational ground states. The lattice holds one molecule per site at a distance of $a_{L}=400 \mathrm{~nm}$, hence, the tunneling between lattice sites is completely suppressed [27]. Considering this low lattice filling, the on-site Hubbard interaction is neglected as well. Thus, the interaction between the molecules is solely determined by the long-range anisotropic dipole-dipole interaction, creating quasi-particles responsible for the excitation transfer along the lattice, the so-called Frenkel excitons [28]. The only two states involved in the dynamics are the ground and the first excited rotational states. The excitation energy operator that models our system is given by [27],

$$
\hat{H}=\sum_{n=1}^{N_{m o l}}\left(B_{e} \hat{N}_{n}^{2}-d_{n} \cdot E\right)+\frac{1}{2} \sum_{n=1}^{N_{m p l}} \sum_{m \neq n}^{N_{m l} l} \hat{V}_{d d}\left(r_{n}-r_{m}\right)
$$

where $\mathbf{r}_{\mathrm{n}}$ is the position of the $n$-th lattice site, $\mathrm{B}_{\mathrm{e}}$ is the rotational constant, $\mathbf{d}_{\mathrm{n}}$ is the electric dipole operator of individual molecules, $\mathbf{E}$ is the constant electric field, $\widehat{N} n$ is the rotational angular momentum operator of the $n$-th molecule and $\widehat{V} d d$ is the dipole-dipole interaction between molecules in different lattice sites. $N_{\text {mol }}$ is the total number of molecules.

The local rotational Hamiltonian, $\sum B_{e} \widehat{N}_{n}^{2}$, corresponds to the crystal energy operator (rotational energy) without intermolecular interactions. In absence of a static electric field, the rotational ground state of the given diatomic molecule has a rotational angular momentum $\mathrm{N}=0$, and the first excited rotational state $\mathrm{N}=1$ is triply degenerated. In the presence of a field $\mathbf{E}$, the Stark effect is accounted for, the degeneracy of $N=1$ is lifted and splits into two sub-energy levels corresponding to the projections $M=0$ and $M= \pm 1$ on the electric field quantization axis. The electric field acts only on the internal coordinates of single molecules, and couples different local molecular states, this coupling depends on the electric field strength. For strong fields beyond a critical value (see reference [29] for critical field theory), the system reduces to a two-level problem considering exclusively $\mathrm{M}=0$ projections. Whereas for a weak static electric field the complete set of internal degrees of freedom must be considered which is the scenario contemplated in the present work.

The dipole-dipole operator, $\hat{V}_{d d}\left(r_{n}-r_{m}\right)$, involves two different sites and mixes two different molecular states, in fact, this interaction determines the dynamics of the system and their features. In the Molecular Fixed Frame (MFF) (commonly used to describe a system of this sort) [30] this operator takes the form,

$$
\hat{V}_{d d}(r)=\frac{1}{3}\left[\hat{d}_{n} \cdot \hat{d}_{m}-3\left(\hat{d}_{n} \cdot \hat{e}\right)\left(\hat{e} \cdot \hat{d}_{m}\right)\right]
$$

Here $\mathbf{r}=\mathbf{r}_{\mathrm{n}}-\mathbf{r}_{\mathrm{m}}$ is the intermolecular vector, $\hat{e}=\vec{r} / r$ is the unit vector in the direction $\mathbf{r}$ and $\hat{d}_{n}, \hat{d}_{m}$ are the dipole moment operators of the molecules at the sites $m$ and $n$ respectively.

The expression (2) does not show how the applied static electric field acts on the long-range interaction [29]. In order to study such effects, it is needed to represent the operator in the Laboratory Fixed Frame (LFF),

$$
\hat{V}_{d d}(r)=-\frac{\sqrt{6}}{r^{3}} \sum_{q=-2}^{2}(-1)^{q} C_{-q}^{2}(r)\left[\hat{d}_{n} \otimes \hat{d}_{m}\right]_{q}^{(2)}
$$

where $C_{-q}^{2}=\sqrt{(4 \pi)} / 5 Y_{-q}^{(2)}(r)$ are the reduced spherical harmonics which describe the movement of the intermolecular axis in the LFF. The one-dimensional confinement of the lattice reduces this coefficient to a simple factor. The operators on the right-hand side in Eq. (3) contain the information about the strength of 
the involved molecular dipole moments. The term can be seen as a second rank tensor product of two tensors of rank one $\widehat{T}_{i}^{1}\left(\hat{d}_{s}\right)$ [31] which represents the dipole moment operator of the molecule located in the site $s \in\{n, m\}$. As a result, we express $\left[\hat{d}_{n} \otimes \hat{d}_{m}\right]_{q}^{(2)}$ as $\sum_{p}\langle p ; q-p \mid 2 q\rangle \widehat{T}_{p}^{1}\left(\hat{d}_{n}\right) \widehat{T}_{q-p}^{1}\left(\hat{d}_{m}\right)$.

By using the second quantization formalism the Hamiltonian (1), that describes collective rotational excitations (excitons), can be written following Ref. [28] as:

$$
\hat{H}_{e x c}=\sum_{n}\left(E_{e g}+D_{n}\right) \hat{P}_{n, M}^{\dagger} \hat{P}_{n, M}+\frac{1}{2} \sum_{n, m \neq n} \sum_{M, M^{\prime}} J_{n, M}^{M, M^{\prime}} \hat{P}_{n, M}^{\dagger} \hat{P}_{m, M^{\prime}}
$$

where the coefficients are given by,

$$
\begin{aligned}
& D_{n}=D_{n}^{e}=\sum_{m \neq n}\left\langle\left\langle e g\left|\hat{V}_{n m}\right| e g\right\rangle-\left\langle g g\left|\hat{V}_{n m}\right| g g\right\rangle\right\} \\
& J_{n m}=J_{n, m}^{M, M^{\prime}}=\left\langle e_{n, M} g_{m}\left|\hat{V}_{n m}\right| g_{n} e_{m, M}\right\rangle .
\end{aligned}
$$

The diagonal term contains the single particle excitation energy, i.e, the energy difference between the excited and ground rotational states, $E_{e g}=E_{e}-E_{g}$, plus the energy shift which is also called gas-condensed matter shift $D_{n}$ due to the interaction between the ground and excited rotational states in neighboring sites (see light-blue solid line in Fig. 1). The second term exhibits the excitation hopping amplitude that accounts for the energy transfer from site $n$ to $m$, the operator $\hat{P}_{n, M}^{i}\left(\hat{P}_{m, M^{\prime}}\right)$ creates (annihilates) a molecule in the site $n(m)$ with a particular degree of freedom $M\left(M^{\prime}\right)$. Specifically, the operators act on the ground state as $\hat{P}_{n, M}^{\dagger}|g\rangle_{n}=|e, M\rangle_{n}$ and like $\hat{P}_{n, M}|e, M\rangle_{m}=|g\rangle_{m}$ on the excited state. Here $|g\rangle_{n}$ and $|e, M\rangle_{n}$ are the fielddressed ground and excited states respectively. The coefficients $J_{n m}$ provide also the molecular selection rules determining the restricted phase-space accessible at low-energies and lead to the coherent and reversible processes that are taking place in the system (see the purple solid line in Fig. 1) plotted for $|e, M\rangle_{n}=|1,0\rangle_{n}$. One can see how the coupling with strong external field reduces the hopping probability for the $M=0$ projection of the excited level while the dipole-dipole interaction between the molecules in the ground state, $\mathrm{V}_{\mathrm{gg}}$, grows from zero to a saturation at large fields (see green curve in Fig. 1).

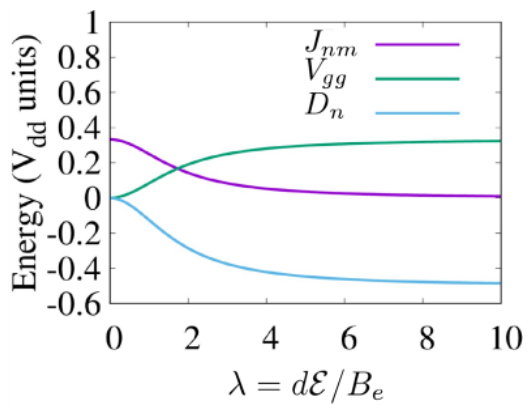

Fig.1. Hamiltonian coefficients, light-blue solid line represents the gas-condensed matter shift $\mathrm{D}_{\mathrm{n}}$. Green solid line is the dipole-dipole interaction between two neighbouring molecules both in the ground rotational state. Purple solid line shows the non-diagonal term $\mathrm{J}_{\mathrm{mn}}$ with $|\boldsymbol{e}, \boldsymbol{M}\rangle_{n}=|\mathbf{1}, \mathbf{0}\rangle_{n}$, i. e. the excitation hopping amplitude between neighbouring sites. All coefficients are measured in units of the dipole-dipole interaction energy and are plotted as a function of the field strength parameter $\boldsymbol{\lambda}=\boldsymbol{d} \boldsymbol{E} / \boldsymbol{B}_{\boldsymbol{e}}$ for a DC field E=Ez. Energy in units of $\boldsymbol{V}_{\boldsymbol{d} \boldsymbol{d}}=\boldsymbol{d}^{2} / \boldsymbol{a}_{\boldsymbol{L}}{ }^{3}$, where $\mathrm{d}$ is the permanent dipole moment, $\boldsymbol{a}_{\boldsymbol{L}}$ the lattice constant and $\mathrm{B}_{\mathrm{e}}$ the rotational constant.

In case the lattice is full of ground-state molecules this term determines the vacuum energy (lack of excitations). Here, we use a DC electric field perpendicular to the molecular arrangement (see Fig. 2.a) leading to the selection rule $\Delta\left(\mathrm{M}_{\mathrm{n}}+\mathrm{M}_{\mathrm{m}}\right)=0, \pm 2$ involving molecules on sites $n$ and $m$ [27]. Finally, working with molecules separated distances greater than $a_{\mathrm{L}}=100 \mathrm{~nm}$ makes the intermolecular interaction considerably smaller compared to the rotational spectrum energy, so it is enough to account only the nearest-neighbor approach.

The Hamiltonian (4) allows us to describe the behavior of the excitations as a function of the external electric field and to study the coherent population transfer between the two-involved rotational molecular levels. We neglect non-linear exciton-exciton interactions, which is a good approximation for a small number of rotational excitations.

\section{2.b. Time evolution calculation}

Numerical calculations reveal important aspects in the ultracold interacting molecular behavior. At low dimensions, quantum fluctuations are enhanced and therefore many-body quantum systems, in general, are 
not susceptible to the standard mean field or perturbation expansion methods so one has to turn to nonperturbative techniques and numerical methods. Here, we use the Matrix Product State (MPS) ansatz [3336] to efficiently approximate the quantum states. This formalism is highly recommended when the amount of entanglement in the system is limited, reducing the calculation complexity from an original exponential to an algebraic growing of the Hilbert space. Based on the MPS state representation, we use the TimeEvolving Block Decimation (TEBD) algorithm [34] to numerically analyze the molecular quantum-chain dynamics, paying special attention to the population transfer and quantum entanglement. The TEBD takes advantage from the fact that the time-evolution operator is approximated using the Trotter-Suzuki expansion formula [37]. Since the entanglement of the system grows on time, keeping the matrices of the same size means to lose information throughout the evolution. In order to avoid so, one has to adapt the value of the entanglement parameter $(\chi)$ every time-step to keep the state as accurate as possible.

We consider a lattice array with $L$ sites which can be separated in two parts one with $l$ sites belonging to a Hilbert space $\mathrm{H}_{B}$ and the other with $L-l$ sites that belongs to $\mathrm{H}_{E}$. Such bipartite system can be separated by applying the Schmidt decomposition [32]. A total state can be written as $|\psi\rangle=\sum \lambda_{\alpha}\left|\alpha_{B}\right\rangle \otimes\left|\alpha_{E}\right\rangle$, where $\left\{\left|\alpha_{B}\right\rangle\right\}$ (on $\mathrm{H}_{B}$ with $\mathrm{N}_{\mathrm{B}}$ the Hilbert dimension) and $\left\{\left|\alpha_{E}\right\rangle\right\}$ (on $\mathrm{H}_{E}$ with $\mathrm{N}_{\mathrm{E}}$ the Hilbert dimension) are two orthonormal basis sets belonging to the respective Hilbert subspace, each with at most $\mathrm{N}_{B}$ basis elements, here $\mathrm{N}_{\mathrm{E}}>\mathrm{N}_{\mathrm{B}}$. The coefficients $\lambda_{\alpha}$ are non-negative real numbers satisfying $\sum \lambda_{\alpha}^{2}=1$, known as the Schmidt coefficients and $\alpha$ runs in the reduced Hilbert subspace. In the numerical implementation, the sum is truncated keeping only $\chi$ states with a truncation error $\sim 10^{-8}$. In a pure state, there is only one density matrix eigenvalue. But the reduced density matrix for a subsystem on $\mathrm{H}_{B}$ is represented by a mixed state $\varrho_{B}=\sum_{\alpha} \lambda_{\alpha}\left|\alpha_{B}\right\rangle \otimes\left|\alpha_{E}\right\rangle$ where the entanglement entropy can be measured using the von Neumann entropy,

$$
S=\sum_{\alpha} \lambda_{\alpha}^{2} \ln \left(\lambda_{\alpha}^{2}\right)
$$

The approximations discussed in sect. 2.1 allow us to use this numerical technique for the time evolution calculation due to the fact that the dipole-dipole interaction can be regarded solely between nearestneighbors [7]. In the following, we consider molecular chains with $L=5$ and $L=10$ sites and initial entanglement parameter $\chi_{0}=8$ reaching $\chi_{\text {final }}=100$, in average, after at most 660 time-steps. In the numerical simulations, the bipartition is performed taking $l=L / 2$, such that for even sites it is exactly at the middle link of the chain whereas for an odd-sites lattice the division is $L / 2$ and $L / 2+1$.

\section{Single molecular-excitation dynamics}

\section{3.a. Two-level system}

In this section, we focus on the dynamics of molecular excitations in the presence of a static and perpendicular electric field (see Fig. 2.a) considering only the $M=0$-projection states, namely $|g\rangle$ and $|e, M=0\rangle$, as shown in Fig. 2.b. The calculations have been done with LiCs alkali molecules following Ref. [38], for which the dipole moment is $\mathrm{d}_{0}=5.523 \mathrm{De}$, the rotational constant is $\mathrm{B}_{\mathrm{e}}=5.816 \mathrm{GHz}$ and the critical electric field for the considered molecule is $\mathrm{E}_{\mathrm{cri}}=2100 \mathrm{~V} / \mathrm{cm}[29]$.

a)

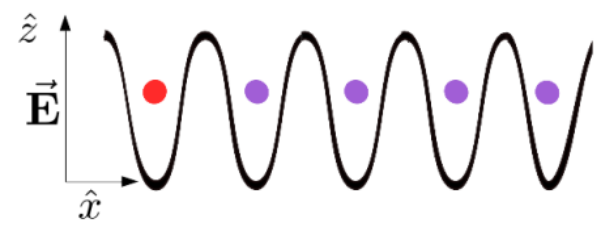

b)

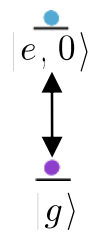

c)

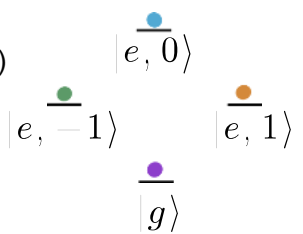

Fig.2. (a) Sketch of the initial state of the evolution, the first site to the left is prepared in one of the excited rotational state projections (red point) whereas the rest of molecules in the lattice are prepared in the rotational ground state (purple points), the DC electric field is located perpendicular to the array. (b) Sketch of the two-level system where only states with $M=0$ projection are taken into account. (c) Sketch of the four-level system where all of the projections are taken into account.

We regard the scenario of small number of rotational excitations, initially keeping only one excitation in the array located at the first lattice site and being the rest of the molecules in the rotational ground-state. The excitation energy transfer in the array in presence of the DC external electric field perpendicular to the lattice is shown in Fig. 3. The evolution of the excitation probability or population in the ground and excited states at the first site is monitored and plotted in Fig 3.a. Initially the population is in the excited state $|e, M=0\rangle$ and coherent oscillations among the two involved states are observed. The envelope of the 
oscillating populations decreases towards the stationary state in the limit of long time evolution to equally distributed population among the two states. The growth of the von Neumann entanglement entropy generated in the time evolution is presented in Fig. 3.b, it oscillates and presents a logarithmic behavior in the limit of long-time evolution as it is expected for the entanglement of 1D systems. The oscillations are caused by the reflection of the excitation on the boundaries due to the small system size. The field strength is $0.5 \mathrm{kV} / \mathrm{cm}$ which is far smaller than the critical field and the lattice constant is $400 \mathrm{~nm}$. For such small fields the presence of the complete four-level system is actually needed, therefore this is considered in the next section.
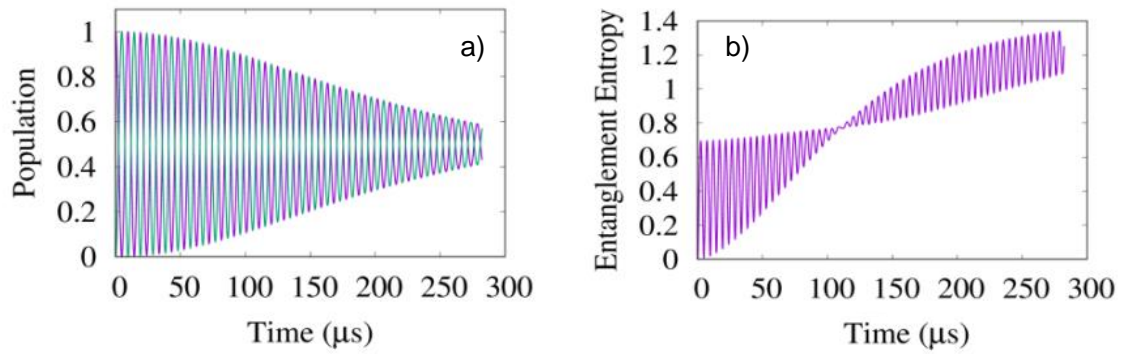

Fig.3. (a) Evolution of the population of the first site (excitation probability) in an array of five LiCs molecules in a DC electric field perpendicular to the array, when the excitation is the state $|\boldsymbol{e}, \boldsymbol{M}=\mathbf{0}\rangle$. (b) Von Neumann entanglement entropy generated in the dynamics. The field strength is $0.5 \mathrm{kV} / \mathrm{cm}$ and the lattice constant is $400 \mathrm{~nm}$.

\section{3.b. Four-level system}

The two-level system is in general the considered scenario in the literature $[4,15,17,27,29,30]$, here our aim is to show that for external electric fields, smaller than the critical value, the full set of internal states must be considered (see Fig. 2.c). Hence, the excitation energy transfer is analysed for several system sizes and initial excitations, the retrieved results are presented in Figs. 4 and 5.

Let us first discuss the case when initially the excited exciton with $M=0$ projection at first site is examined. Figure 4.a shows the evolution of the energy transfer for a chain with L=10 sites, the population at the monitored site smoothly evolves for short time scales. Here, we present the dynamics of the first $15 \mu \mathrm{s}$ (time scale in units of $1 / \mathrm{V}_{\mathrm{dd}}$ ), and it can be observed that all molecular states get populated at such short time. The corresponding von Neumann entanglement entropy is shown in Fig. 4.c. The behaviour is monotonic and the sharp initial growth ceases after $5 \mu$ s followed by a growth with less slope.
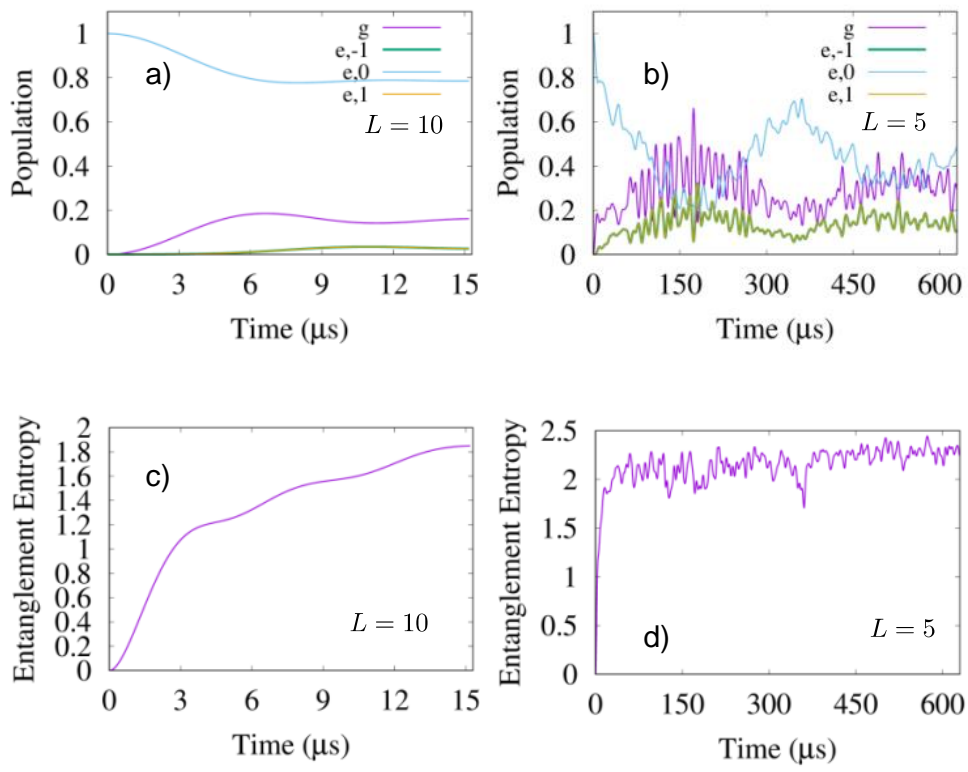

Fig.4. Evolution of the population of the first site in an array of (a) ten LiCs molecules and (b) five molecules in a DC ele ctric field perpendicular to the array, when the excitation is in the projection $M=0$. Von Neumann entanglement entropy generated in the dynamics with (c) ten molecules and (d) five molecules. The field strength is $0.5 \mathrm{kV} / \mathrm{cm}$ and the lattice constant is $400 \mathrm{~nm}$. Time scale in units of $1 / V_{d d}$. In panels (a) and (b) the curves for $|\boldsymbol{e}, \mathbf{\pm 1}\rangle$ are one on top of the other. 
For shorter chains ( $\mathrm{L}=5$ sites) the evolution can be simulated for longer times as it is shown in Fig. 4.b where the population dynamics is presented up to $600 \mu \mathrm{s}$. This long-term evolution reveals a collapse and revival of the excitation probability to have the molecule in its initial projection with a return period of approximately $400 \mu \mathrm{s}$. Even more, at this precise time the entanglement entropy (Fig. 4.d) presents a sharp valley because the system gets closest to the initial uncorrelated state. It can also be mentioned that the population in the $M= \pm 1$ excited-projections have non-trivial dynamics showing the importance of considering them in the on-site basis to simulate faithfully the excitation energy transfer. Additionally, it is worth noticing that the von Neumann entropy saturates in the steady state, exhibiting the finite entanglement that can be generated in 1D quantum systems.

Finally, when the $|e, 1\rangle$ state is taken to be the initial state (similar analysis applies for $|e,-1\rangle$ ), see Fig. 5.a, the population rapidly drops into $|g\rangle$ state, then goes into $|e,-1\rangle$ and at last the $|e, 0\rangle$ enters in the dynamics. The field dressed states with $M= \pm 1$ have the same energy, hence, they have the same probability to be populated, being the reason why $|e,-1\rangle$ state comes first in the dynamics rather than $|e, 0\rangle$ state. Highamplitude coherent oscillations take place for this initial condition and the considered evolution time. The entanglement of the energy transfer is presented in Fig. 5.b, it increases monotonically towards a saturation and presents a similar behaviour as Fig 4.c.
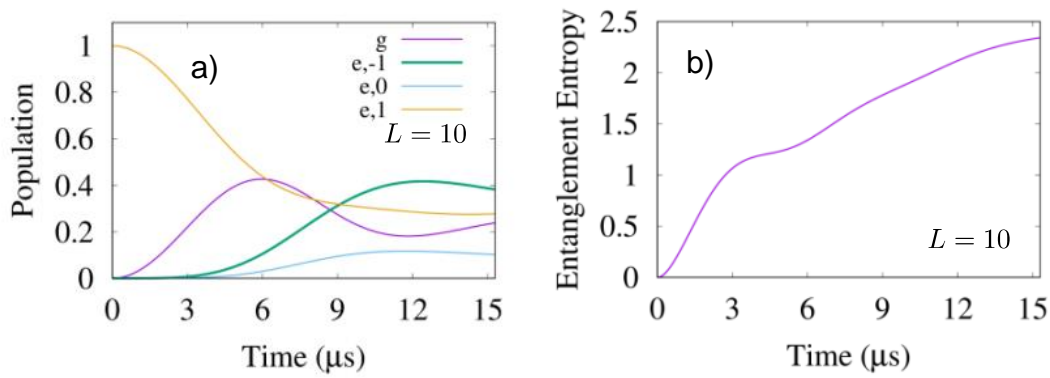

Fig.5. (Color online) (a) Evolution of the population of the firs site in an array of ten LiCs molecules when the excitation is the projection $\boldsymbol{M}=$ 1. (b) Von Neumann entanglement entropy generated in the time-evolution. The field strength is $0.5 \mathrm{kV} / \mathrm{cm}$ and the lattice constant is $400 \mathrm{~nm}$.

\section{Conclusions}

We studied a LiCs molecular gas loaded on an 1D optical lattice prepared in the strongly-correlated Mottinsulator regime with one molecule per lattice site. The molecules are in the lowest electronic and vibrational state and the coherent population transfer between the internal rotational states as a function of an external DC electric field is analyzed. Due to the large intermolecular lattice distance and low filling, the hopping between nearest-neighbors and the on-site Hubbard interaction are suppressed, hence, the dipole-dipole interaction in the nearest-neighbor approximation solely governs the dynamics of the excitations. In general, the two-level approximation is the considered scenario in the literature, here we show that for field strengths lower than the critical field the full set of internal states must be considered and the $M= \pm 1$ states have non-trivial dynamics. Coherent transfer of population among the field-dressed states along the lattice is shown for several system sizes and initial conditions. Our numerical simulations show that for weak fields the short-term evolution presents a continuously growth of the von Neumann entropy but at the long term it saturates as it is expected for 1D quantum systems. For few lattice sites the evolution can take place for a considerable long time scale of the order of $10^{-3} \mathrm{~s}$, enough time to visualize a collapse and revival of the initial exciton population, finding a sharp valley in the entanglement at the time when the initial exciton projection reaches its maximal probability again.

This kind of studies allow us to determine the more suitable set of internal states to describe faithfully the quantum dynamics taking place in these many-body periodic systems.

\section{Acknowledgements}

This work has been supported by Universidad del Valle under the internal projects 71107 and the Colombian Science, Technology and Innovation Fundation--COLCIENCIAS "Francisco José de Caldas" under project 110665842793 (contract FP-005-2015). The authors acknowledge the Excellence Center of New Materials (CENM) Gran no. RC275-2011 for financial support of the research group. 